\title{
New results on higher-order Daehee and Bernoulli numbers and polynomials
}

\author{
Beih S El-Desouky* and Abdelfattah Mustafa
}

"Correspondence:

b_eldesouky@yahoo.com

Department of Mathematics,

Faculty of Science, Mansoura

University, Mansoura, 35516, Egypt

\begin{abstract}
We derive a new matrix representation for higher-order Daehee numbers and polynomials, higher-order $\lambda$-Daehee numbers and polynomials, and twisted $\lambda$-Daehee numbers and polynomials of order $k$. This helps us to obtain simple and short proofs of many previous results on higher-order Daehee numbers and polynomials. Moreover, we obtain recurrence relations, explicit formulas, and some new results for these numbers and polynomials. Furthermore, we investigate the relation between these numbers and polynomials and Stirling, Nörlund, and Bernoulli numbers of higher-order. Some numerical results and program are introduced using Mathcad for generating higher-order Daehee numbers and polynomials. The results of this article generalize the results derived very recently by El-Desouky and Mustafa (Appl. Math. Sci. 9(73):3593-3610, 2015).
\end{abstract}

MSC: Primary 05A19; secondary 11C20; 11B73; 11 T06

Keywords: Daehee numbers; Daehee polynomials; higher-order Daehee numbers; higher-order Daehee polynomials; higher-order Bernoulli polynomials; matrix representation

\section{Introduction}

For $\alpha \in \mathbb{N}$, the Bernoulli polynomials of order $\alpha$ are defined by (see [1-15])

$$
\left(\frac{t}{e^{t}-1}\right)^{\alpha} e^{x t}=\sum_{n=0}^{\infty} B_{n}^{(\alpha)}(x) \frac{t^{n}}{n !}
$$

When $x=0, B_{n}^{(\alpha)}=B_{n}^{(\alpha)}(0)$ are the Bernoulli numbers of order $\alpha$ defined by

$$
\left(\frac{t}{e^{t}-1}\right)^{\alpha}=\sum_{n=0}^{\infty} B_{n}^{(\alpha)} \frac{t^{n}}{n !} .
$$

The Daehee polynomials are defined by (see $[11,12]$ and $[15])$

$$
\left(\frac{\log (1+t)}{t}\right)(1+t)^{x}=\sum_{n=0}^{\infty} D_{n}(x) \frac{t^{n}}{n !} .
$$

In the special case $x=0, D_{n}=D_{n}(0)$ are called the Daehee numbers defined by

$$
\left(\frac{\log (1+t)}{t}\right)=\sum_{n=0}^{\infty} D_{n} \frac{t^{n}}{n !}
$$

(c) 2016 El-Desouky and Mustafa. This article is distributed under the terms of the Creative Commons Attribution 4.0 International License (http://creativecommons.org/licenses/by/4.0/), which permits unrestricted use, distribution, and reproduction in any medium, provided you give appropriate credit to the original author(s) and the source, provide a link to the Creative Commons license, and indicate if changes were made. 
The Stirling numbers of the first and second kind are defined, respectively, by

$$
(x)_{n}=\prod_{i=0}^{n-1}(x-i)=\sum_{l=0}^{n} s_{1}(n, l) x^{l},
$$

where $s_{1}(n, 0)=\delta_{n, 0}, s_{1}(n, k)=0$, for $k>n$, and

$$
x^{n}=\sum_{k=0}^{n} s_{2}(n, k)(x)_{k}
$$

where $s_{2}(n, 0)=\delta_{n, 0}, s_{2}(n, k)=0$ for $k>n$, and $\delta_{n, k}$ is the Kronecker delta.

The Stirling numbers of the second kind have the generating function (see $[2,3,5,6]$ and [7])

$$
\left(e^{t}-1\right)^{m}=m ! \sum_{l=m}^{\infty} s_{2}(l, m) \frac{t^{l}}{l !} .
$$

\section{Higher-order Daehee numbers and polynomials}

In this section, we derive explicit formulas and recurrence relations for the higher-order Daehee numbers and polynomials of the first and second kinds. Also, we give a relation between these numbers and Nörlund numbers. Furthermore, we introduce the matrix representation of some results for higher-order Daehee numbers and polynomials obtained by Kim et al. [8] in terms of Stirling numbers, Nörlund numbers, and Bernoulli numbers of higher order and give simple and short proofs of these results.

Kim et al. [8] defined the Daehee numbers of the first kind of order $k$ by the generating function

$$
\sum_{n=0}^{\infty} D_{n}^{(k)} \frac{t^{n}}{n !}=\left(\frac{\log (1+t)}{t}\right)^{k}
$$

An explicit formula for $D_{n}^{(k)}$ is given by the following theorem.

Theorem 1 For $n \in \mathbb{Z}, k \in \mathbb{N}$, we have

$$
D_{n}^{(k)}=n ! \sum_{l_{1}+l_{2}+\cdots+l_{k}=n+k} \frac{(-1)^{n}}{l_{1} l_{2} \cdots l_{k}}
$$

Proof From Eq. (8) we have

$$
\sum_{n=0}^{\infty} D_{n}^{(k)} \frac{t^{n+k}}{n !}=(\log (1+t))^{k}=\left(\sum_{l=1}^{\infty} \frac{(-1)^{l-1}}{l} t^{l}\right)^{k} .
$$

Using the Cauchy rule for a product of series, we obtain

$$
\sum_{n=0}^{\infty} D_{n}^{(k)} \frac{t^{n+k}}{n !}=\sum_{r=k}^{\infty} \sum_{l_{1}+l_{2}+\cdots+l_{k}=r}^{\infty} \frac{(-1)^{r-k}}{l_{1} l_{2} \cdots l_{k}} t^{r} .
$$


Letting $r-k=n$ in the right-hand side, we have

$$
\sum_{n=0}^{\infty} D_{n}^{(k)} \frac{t^{n+k}}{n !}=\sum_{n=0}^{\infty} \sum_{l_{1}+l_{2}+\cdots+l_{k}=n+k}^{\infty} \frac{(-1)^{n}}{l_{1} l_{2} \cdots l_{k}} t^{n+k} .
$$

Equating the coefficients of $t^{n+k}$ on both sides yields (9). This completes the proof.

Remark 1 It is worth noting that setting $k=1$ in (9), we get Eq. (2.2) of [1] as a particular case.

Kim et al. ([8], Theorem 1) proved that (see [16]), for $n \in \mathbb{Z}$ and $k \in \mathbb{N}$,

$$
D_{n}^{(k)}=\frac{s_{1}(n+k, k)}{\left(\begin{array}{c}
n+k \\
k
\end{array}\right)} .
$$

We can represent the Daehee numbers of the first kind of order $k$ by an $(n+1) \times(k+1)$ matrix, $0 \leq k \leq n$, as follows:

$$
\mathbf{D}^{(k)}=\left(\begin{array}{ccccc}
D_{0}^{(0)} & D_{0}^{(1)} & D_{0}^{(2)} & \ldots & D_{0}^{(k)} \\
D_{1}^{(0)} & D_{1}^{(1)} & D_{1}^{(2)} & \ldots & D_{1}^{(k)} \\
\vdots & \vdots & \vdots & \ddots & \vdots \\
D_{n}^{(0)} & D_{n}^{(1)} & D_{n}^{(2)} & \ldots & D_{n}^{(k)}
\end{array}\right) .
$$

The following theorem gives a recurrence relation for Daehee numbers of the first kind of order $k$.

Theorem 2 For $n \in \mathbb{Z}$ and $k \in \mathbb{N}$, we have

$$
D_{n+1}^{(k)}=\frac{1}{n+k+1}\left[k D_{n+1}^{(k-1)}-(n+1)(n+k) D_{n}^{(k)}\right],
$$

where $D_{0}^{(k)}=1, D_{n}^{(0)}=0$ for $n \geq 1, k=0,1, \ldots, n$.

Proof The recurrence relation for the Stirling numbers of the first kind is given by

$$
s_{1}(n+1, k)=s_{1}(n, k-1)-n s_{1}(n, k) .
$$

Replacing $n$ by $n+k$, we get

$$
s_{1}(n+k+1, k)=s_{1}(n+k, k-1)-(n+k) s_{1}(n+k, k) .
$$

By using relation (10) we have

$$
\left(\begin{array}{c}
n+k+1 \\
k
\end{array}\right) D_{n+1}^{(k)}=\left(\begin{array}{c}
n+k \\
k-1
\end{array}\right) D_{n+1}^{(k-1)}-(n+k)\left(\begin{array}{c}
n+k \\
k
\end{array}\right) D_{n}^{(k)} ;
$$

hence,

$$
(n+k+1) D_{n+1}^{(k)}=k D_{n+1}^{(k-1)}-(n+k)(n+1) D_{n}^{(k)} .
$$

This completes the proof. 
A Mathcad program is written and executed to generate the higher-order Daehee numbers using the recurrence relation (11); see the Appendix.

For example, if $0 \leq n \leq 3$ and $0 \leq k \leq n$, then we have

$$
\mathbf{D}^{(k)}=\left(\begin{array}{cccc}
1 & 1 & 1 & 1 \\
0 & -1 / 2 & -1 & -3 / 2 \\
0 & 2 / 3 & 11 / 6 & 7 / 2 \\
0 & -3 / 2 & -5 & -45 / 4
\end{array}\right)
$$

Kim et al. ([8], Theorem 4) proved the following result: for $n \in \mathbb{Z}$ and $k \in \mathbb{N}$,

$$
B_{n}^{(k)}=\sum_{m=0}^{n} D_{m}^{(k)} s_{2}(n, m) .
$$

Remark 2 We can write this relation in the matrix form as follows:

$$
\mathbf{B}^{(k)}=\mathbf{S}_{2} \mathbf{D}^{(k)},
$$

where $\mathbf{D}^{(k)}$ is an $(n+1) \times(k+1)$ matrix $(0 \leq k \leq n)$ for the Daehee numbers of the first kind of order $k$, and $\mathbf{S}_{2}$ is an $(n+1) \times(n+1)$ lower triangular matrix for the Stirling numbers of the second kind, and $\mathbf{B}^{(k)}$ is an $(n+1) \times(k+1)$ matrix for the Bernoulli numbers of order $k$.

For example, setting $0 \leq n \leq 3$ and $0 \leq k \leq n$ in (13), we have

$$
\left(\begin{array}{cccc}
1 & 0 & 0 & 0 \\
0 & 1 & 0 & 0 \\
0 & 1 & 1 & 0 \\
0 & 1 & 3 & 1
\end{array}\right)\left(\begin{array}{cccc}
1 & 1 & 1 & 1 \\
0 & -1 / 2 & -1 & -3 / 2 \\
0 & 2 / 3 & 11 / 6 & 7 / 2 \\
0 & -3 / 2 & -5 & -45 / 4
\end{array}\right)=\left(\begin{array}{cccc}
1 & 1 & 1 & 1 \\
0 & -1 / 2 & -1 & -3 / 2 \\
0 & 1 / 6 & 5 / 6 & 2 \\
0 & 0 & -1 / 2 & -9 / 4
\end{array}\right)
$$

Kim et al. ([8], Theorem 3) introduced the following result: for $n \in \mathbb{Z}$ and $k \in \mathbb{N}$,

$$
D_{n}^{(k)}=\sum_{m=0}^{n} s_{1}(n, m) B_{m}^{(k)}
$$

We can write this relation in the matrix form as follows:

$$
\mathbf{D}^{(k)}=\mathbf{S}_{1} \mathbf{B}^{(k)},
$$

where $\mathbf{S}_{1}$ is an $(n+1) \times(n+1)$ lower triangular matrix for the Stirling numbers of the first kind.

For example, setting $0 \leq n \leq 3$ and $0 \leq k \leq n$ in (15), we have

$$
\left(\begin{array}{cccc}
1 & 0 & 0 & 0 \\
0 & 1 & 0 & 0 \\
0 & -1 & 1 & 0 \\
0 & 2 & -3 & 1
\end{array}\right)\left(\begin{array}{cccc}
1 & 1 & 1 & 1 \\
0 & -1 / 2 & -1 & -3 / 2 \\
0 & 1 / 6 & 5 / 6 & 2 \\
0 & 0 & -1 / 2 & -9 / 4
\end{array}\right)=\left(\begin{array}{cccc}
1 & 1 & 1 & 1 \\
0 & -1 / 2 & -1 & -3 / 2 \\
0 & 2 / 3 & 11 / 6 & 7 / 2 \\
0 & -3 / 2 & -5 & -45 / 4
\end{array}\right) .
$$


Remark 3 Using the matrix form (15), we easily derive a short proof of Theorem 4 in Kim et al. [8]. Multiplying both sides by the Stirling number of second kind, we get

$$
\mathbf{S}_{2} \mathbf{D}^{(k)}=\mathbf{S}_{2} \mathbf{S}_{1} \mathbf{B}^{(k)}=\mathbf{I} \mathbf{B}^{(k)}=\mathbf{B}^{(k)},
$$

where $\mathbf{I}$ is the identity matrix of order $(n+1)$.

Kim et al. [8] defined the Daehee polynomials of order $k$ by the generating function as follows:

$$
\sum_{n=0}^{\infty} D_{n}^{(k)}(x) \frac{t^{n}}{n !}=\left(\frac{\log (1+t)}{t}\right)^{k}(1+t)^{x} .
$$

Liu and Srivastava [14] defined the Nörlund numbers of the second kind $b_{n}^{(x)}$ as follows:

$$
\left(\frac{t}{\log (1+t)}\right)^{x}=\sum_{n=0}^{\infty} b_{n}^{(x)} t^{n}
$$

Next, we give a relation between the Daehee polynomials of order $k$ and the Nörlund numbers of the second kind $b_{n}^{(x)}$ in the following theorem.

Theorem 3 For $m \in \mathbb{Z}$ and $k \in \mathbb{N}$, we have

$$
D_{m}^{(k)}(z)=m ! \sum_{n=0}^{m}\left(\begin{array}{c}
z \\
m-n
\end{array}\right) b_{n}^{(-k)}
$$

Proof From Eq. (17) by multiplying both sides by $(1+t)^{z}$ we have

$$
\begin{aligned}
\left(\frac{t}{\log (1+t)}\right)^{x}(1+t)^{z} & =\sum_{n=0}^{\infty} b_{n}^{(x)} t^{n}(1+t)^{z}=\sum_{n=0}^{\infty} b_{n}^{(x)} t^{n} \sum_{i=0}^{\infty}\left(\begin{array}{c}
z \\
i
\end{array}\right) t^{i} \\
& =\sum_{n=0}^{\infty} \sum_{m=n}^{\infty} b_{n}^{(x)}\left(\begin{array}{c}
z \\
m-n
\end{array}\right) t^{m}=\sum_{m=0}^{\infty} \sum_{n=0}^{m}\left(\begin{array}{c}
z \\
m-n
\end{array}\right) b_{n}^{(x)} t^{m}
\end{aligned}
$$

Replacing $x$ by $-k$ in (19), we have

$$
\begin{aligned}
\left(\frac{\log (1+t)}{t}\right)^{k}(1+t)^{z} & =\sum_{m=0}^{\infty} \sum_{n=0}^{m}\left(\begin{array}{c}
z \\
m-n
\end{array}\right) b_{n}^{(-k)} t^{m} \\
& =\sum_{m=0}^{\infty} m ! \sum_{n=0}^{m}\left(\begin{array}{c}
z \\
m-n
\end{array}\right) b_{n}^{(-k)} \frac{t^{m}}{m !}
\end{aligned}
$$

From (16) and (20) we have (18). This completes the proof.

Corollary 1 Setting $k=1$ in (18), we have

$$
D_{m}(z)=m ! \sum_{n=0}^{m}\left(\begin{array}{c}
z \\
m-n
\end{array}\right) b_{n}^{(-1)} .
$$


Setting $z=0$ in (18), we have the following relation between Daehee numbers of higher order and Nörlund numbers of the second kind.

Corollary 2 For $k \in \mathbb{N}$, by setting $z=0$ in (18) we obtain

$$
D_{m}^{(k)}=m ! b_{m}^{(-k)}
$$

The following relation between Bernoulli numbers and Bernoulli polynomials of order $k$ is given by Kimura [13]:

$$
B_{n}^{(k)}(x)=\sum_{j=0}^{n}\left(\begin{array}{l}
n \\
j
\end{array}\right) B_{j}^{(k)} x^{n-j}
$$

Therefore, we can represent (23) in the matrix form

$$
\mathbf{B}^{(k)}(x)=\mathbf{P}(x) \mathbf{B}^{(k)},
$$

where $\mathbf{B}^{(k)}(x)$ is an $(n+1) \times(k+1)$ matrix $(0 \leq k \leq n)$ for Bernoulli polynomials of order $k$,

$$
\mathbf{B}^{(k)}(x)=\left(\begin{array}{ccccc}
B_{0}^{(0)}(x) & B_{0}^{(1)}(x) & B_{0}^{(2)}(x) & \cdots & B_{0}^{(k)}(x) \\
B_{1}^{(0)}(x) & B_{1}^{(1)}(x) & B_{1}^{(2)}(x) & \cdots & B_{1}^{(k)}(x) \\
\vdots & \vdots & \vdots & \ddots & \vdots \\
B_{n}^{(0)}(x) & B_{n}^{(1)}(x) & B_{n}^{(2)}(x) & \cdots & B_{n}^{(k)}(x)
\end{array}\right)
$$

where the column $k$ represents the Bernoulli polynomials of order $k, \mathbf{B}^{(k)}$ is an $(n+1) \times$ $(k+1)$ matrix $(0 \leq k \leq n)$ for Bernoulli numbers of order $k$, and the matrix $\mathbf{P}(x)$, the Pascal matrix, is the $(n+1) \times(n+1)$ lower triangular matrix defined by

$$
(\mathbf{P}(x))_{i j}=\left\{\begin{array}{ll}
\left(\begin{array}{l}
i \\
j
\end{array}\right) x^{i-j}, & i \geq j, \\
0 & \text { otherwise, }
\end{array} \quad i, j=0,1, \ldots, n .\right.
$$

For example, setting $0 \leq n \leq 3$ and $0 \leq k \leq n$ in (24), we have

$$
\begin{aligned}
\left(\begin{array}{cccc}
1 & 0 & 0 & 0 \\
x & 1 & 0 & 0 \\
x^{2} & 2 x & 1 & 0 \\
x^{3} & 3 x^{2} & 3 x & 1
\end{array}\right)\left(\begin{array}{cccc}
1 & 1 & 1 & 1 \\
0 & -1 / 2 & -1 & -3 / 2 \\
0 & 1 / 6 & 5 / 6 & 2 \\
0 & 0 & -1 / 2 & -9 / 4
\end{array}\right) \\
=\left(\begin{array}{cccc}
1 & 1 & 1 & 1 \\
x & x-\frac{1}{2} & x-1 & x-\frac{3}{2} \\
x^{2} & x^{2}-x+\frac{1}{6} & x^{2}-2 x+\frac{5}{6} & x^{2}-3 x+2 \\
x^{3} & x^{3}-\frac{3}{2} x^{2}+\frac{1}{2} x & x^{3}-3 x^{2}+\frac{5}{2} x-\frac{1}{2} & x^{3}-\frac{9}{2} x^{2}+6 x-\frac{9}{4}
\end{array}\right) .
\end{aligned}
$$

Kim et al. ([8], Theorem 5) introduced the following result: for $n \in \mathbb{Z}$ and $k \in \mathbb{N}$,

$$
D_{n}^{(k)}(x)=\sum_{m=0}^{n} s_{1}(n, m) B_{m}^{(k)}(x) .
$$


We can write this relation in the matrix form as follows:

$$
\mathbf{D}^{(k)}(x)=\mathbf{S}_{1} \mathbf{B}^{(k)}(x),
$$

where $\mathbf{D}^{(k)}(x)$ is the $(n+1) \times(k+1)$ matrix for the Daehee polynomials of the first kind with order $k$, and $\mathbf{B}^{(k)}(x)$ is the $(n+1) \times(k+1)$ matrix for the Bernoulli polynomials of order $k$.

For example, setting $0 \leq n \leq 3$ and $0 \leq k \leq n$ in (26), we have

$$
\begin{aligned}
& \left(\begin{array}{cccc}
1 & 0 & 0 & 0 \\
0 & 1 & 0 & 0 \\
0 & -1 & 1 & 0 \\
0 & 2 & -3 & 1
\end{array}\right) \\
& \quad\left(\begin{array}{ccccc}
1 & 1 & 1 & 1 \\
x & x-\frac{1}{2} & x-1 & x-\frac{3}{2} \\
x^{2} & x^{2}-x+\frac{1}{6} & x^{2}-2 x+\frac{5}{6} & x^{2}-3 x+2 \\
x^{3} & x^{3}-\frac{3}{2} x^{2}+\frac{1}{2} x & x^{3}-3 x^{2}+\frac{5}{2} x-\frac{1}{2} & x^{3}-\frac{9}{2} x^{2}+6 x-\frac{9}{4}
\end{array}\right) \\
& =\left(\begin{array}{cccc}
x^{2} & 1 & 1 & x-\frac{3}{2} \\
x^{3}-3 x^{2}+2 x & x^{3}-\frac{9}{2} x^{2}+\frac{11}{2} x-\frac{3}{2} & x^{3}-6 x^{2}+\frac{21}{2} x-5 & x^{3}-\frac{15}{2} x^{2}+17 x-\frac{45}{4}
\end{array}\right) .
\end{aligned}
$$

$\operatorname{Kim}$ et al. ([8], Theorem 7) introduced the following result: for $n \in \mathbb{Z}$ and $k \in \mathbb{N}$,

$$
B_{n}^{(k)}(x)=\sum_{m=0}^{n} D_{m}^{(k)}(x) s_{2}(n, m) .
$$

We can write Eq. (27) in the matrix form as follows:

$$
\mathbf{B}^{(k)}(x)=\mathbf{S}_{2} \mathbf{D}^{(k)}(x) .
$$

For example, setting $0 \leq n \leq 3$ and $0 \leq k \leq n$ in (28), we have

$$
\begin{aligned}
& \left(\begin{array}{llll}
1 & 0 & 0 & 0 \\
0 & 1 & 0 & 0 \\
0 & 1 & 1 & 0 \\
0 & 1 & 3 & 1
\end{array}\right) \\
& \times\left(\begin{array}{cccc}
1 & 1 & 1 & 1 \\
x & x-\frac{1}{2} & x-1 & x-\frac{3}{2} \\
x^{2}-x & x^{2}-2 x+\frac{2}{3} & x^{2}-3 x+\frac{11}{6} & x^{2}-4 x+\frac{7}{2} \\
x^{3}-3 x^{2}+2 x & x^{3}-\frac{9}{2} x^{2}+\frac{11}{2} x-\frac{3}{2} & x^{3}-6 x^{2}+\frac{21}{2} x-5 & x^{3}-\frac{15}{2} x^{2}+17 x-\frac{45}{4}
\end{array}\right) \\
& =\left(\begin{array}{cccc}
1 & 1 & 1 & 1 \\
x & x-\frac{1}{2} & x-1 & x-\frac{3}{2} \\
x^{2} & x^{2}-x+\frac{1}{6} & x^{2}-2 x+\frac{5}{6} & x^{2}-3 x+2 \\
x^{3} & x^{3}-\frac{3}{2} x^{2}+\frac{1}{2} x & x^{3}-3 x^{2}+\frac{5}{2} x-\frac{1}{2} & x^{3}-\frac{9}{2} x^{2}+6 x-\frac{9}{4}
\end{array}\right) .
\end{aligned}
$$


Remark 4 We can prove Theorem 7 in Kim et al. [8] by using the matrix form (26) as follows. Multiplying both sides of (26) by the Stirling number of second kind, we have

$$
\mathbf{S}_{2} \mathbf{D}^{(k)}(x)=\mathbf{S}_{2} \mathbf{S}_{1} \mathbf{B}^{(k)}(x)=\mathbf{I} \mathbf{B}^{(k)}(x)=\mathbf{B}^{(k)}(x) .
$$

Kim et al. [8] defined the Daehee numbers of the second kind of order $k$ by the generating function as follows:

$$
\sum_{n=0}^{\infty} \hat{D}_{n}^{k}[(k)] \frac{t^{n}}{n !}=\left(\frac{(1-t) \log (1-t)}{-t}\right)^{k}
$$

Kim et al. ([8], Theorem 8) introduced the following result: for $n \in \mathbb{Z}$ and $k \in \mathbb{N}$,

$$
\hat{D}_{n}^{k}[(k)]=\sum_{l=0}^{n}\left[\begin{array}{l}
n \\
l
\end{array}\right] B_{l}^{(k)}
$$

where $\left[\begin{array}{l}n \\ l\end{array}\right]=(-1)^{n-l} s_{1}(n, l)=\left|s_{1}(n, k)\right|=\mathfrak{s}(n, k)$, and $\mathfrak{s}(n, k)$ are the signless Stirling numbers of the first kind; see [3] and [5, 6].

We can write this theorem in the matrix form as follows:

$$
\hat{\mathbf{D}}^{(k)}=\mathfrak{S B}^{(k)},
$$

where $\hat{\mathbf{D}}^{(k)}$ is the $(n+1) \times(k+1)$ matrix of Daehee numbers of the second kind of order $k$, and $\mathfrak{S}$ is the $(n+1) \times(n+1)$ lower triangular matrix for the signless Stirling numbers of the first kind.

For example, setting $0 \leq n \leq 3$ and $0 \leq k \leq n$ in (31), we have

$$
\left(\begin{array}{llll}
1 & 0 & 0 & 0 \\
0 & 1 & 0 & 0 \\
0 & 1 & 1 & 0 \\
0 & 2 & 3 & 1
\end{array}\right)\left(\begin{array}{cccc}
1 & 1 & 1 & 1 \\
0 & -1 / 2 & -1 & -3 / 2 \\
0 & 1 / 6 & 5 / 6 & 2 \\
0 & 0 & -1 / 2 & -9 / 4
\end{array}\right)=\left(\begin{array}{cccc}
1 & 1 & 1 & 1 \\
0 & -1 / 2 & -1 & -3 / 2 \\
0 & -1 / 3 & -1 / 6 & 1 / 2 \\
0 & -1 / 2 & 0 & 3 / 4
\end{array}\right)
$$

Kim et al. ([8], Theorem 9) introduced the following result: for $n \in \mathbb{Z}$ and $k \in \mathbb{N}$,

$$
B_{n}^{(k)}=\sum_{m=0}^{n}(-1)^{n-m} s_{2}(n, m) \hat{D}_{m}^{k}[(k)]
$$

We can write Eq. (32) in the matrix form as follows:

$$
\mathbf{B}^{(k)}=\tilde{\mathbf{S}}_{2} \hat{\mathbf{D}}^{(k)}
$$

where $\tilde{\mathbf{S}}_{2}$ is the $(n+1) \times(n+1)$ lower triangular matrix for signed Stirling numbers of the second kind defined by

$$
\left(\tilde{\mathbf{S}}_{2}\right)_{i j}=\left\{\begin{array}{ll}
(-1)^{i-j} s_{2}(i, j), & i \geq j, \\
0 & \text { otherwise, }
\end{array} \quad i, j=0,1, \ldots, n .\right.
$$


For example, setting $0 \leq n \leq 3$ and $0 \leq k \leq n$ in (33), we have

$$
\left(\begin{array}{cccc}
1 & 0 & 0 & 0 \\
0 & 1 & 0 & 0 \\
0 & -1 & 1 & 0 \\
0 & 1 & -3 & 1
\end{array}\right)\left(\begin{array}{cccc}
1 & 1 & 1 & 1 \\
0 & -1 / 2 & -1 & -3 / 2 \\
0 & -1 / 3 & -1 / 6 & 1 / 2 \\
0 & -1 / 2 & 0 & 3 / 4
\end{array}\right)=\left(\begin{array}{cccc}
1 & 1 & 1 & 1 \\
0 & -1 / 2 & -1 & -3 / 2 \\
0 & 1 / 6 & 5 / 6 & 2 \\
0 & 0 & -1 / 2 & -9 / 4
\end{array}\right)
$$

Remark 5 We can prove Theorem 9 in Kim et al. [8] by using the matrix form (31) as follows. Multiplying both sides of (31) by the matrix of sign Stirling numbers of second kind $\tilde{\mathbf{S}}_{2}$, we have

$$
\tilde{\mathbf{S}}_{2} \hat{\mathbf{D}}^{(k)}=\tilde{\mathbf{S}}_{2} \boldsymbol{S} \mathbf{B}^{(k)}=\mathbf{I} \mathbf{B}^{(k)}=\mathbf{B}^{(k)},
$$

which gives Eq. (33), where we used the identity $\tilde{\mathbf{S}}_{2} \mathfrak{S}=\mathbf{I}$.

Kim et al. [8] defined the Daehee polynomials of the second kind of order $k$ by the generating function as follows:

$$
\sum_{n=0}^{\infty} \hat{D}_{n}^{k}[(k)](x) \frac{t^{n}}{n !}=\left(\frac{(1-t) \log (1-t)}{-t}\right)^{k}(1-t)^{x}
$$

Kim et al. ([8], Eq. (31)) introduced the following result: for $n \in \mathbb{Z}$ and $k \in \mathbb{N}$,

$$
\hat{D}_{n}^{k}[(k)](x)=\sum_{m=0}^{n}(-1)^{n-m} s_{1}(n, m) B_{m}^{(k)}(-x) .
$$

Equation (35) is equivalent to

$$
\hat{D}_{n}^{k}[(k)](x)=\sum_{m=0}^{n} \mathfrak{s}(n, m) B_{m}^{(k)}(-x) .
$$

We can write Eq. (36) in the matrix form as follows:

$$
\hat{\mathbf{D}}^{(k)}(x)=\mathfrak{S B}^{(k)}(-x),
$$

where $\hat{\mathbf{D}}^{(k)}(x)$ is the $(n+1) \times(k+1)$ matrix of the Daehee polynomials of the second kind of order $k$, and $\mathbf{B}^{(k)}(x)$ is the $(n+1) \times(k+1)$ matrix of the Bernoulli polynomials.

For example, setting $0 \leq n \leq 3$ and $0 \leq k \leq n$ in (37), we have

$$
\begin{aligned}
& \left(\begin{array}{llll}
1 & 0 & 0 & 0 \\
0 & 1 & 0 & 0 \\
0 & 1 & 1 & 0 \\
0 & 2 & 3 & 1
\end{array}\right)\left(\begin{array}{cccc}
1 & 1 & 1 & 1 \\
-x & -x-\frac{1}{2} & -x-1 & -x-\frac{3}{2} \\
x^{2} & x^{2}+x+\frac{1}{6} & x^{2}+2 x+\frac{5}{6} & x^{2}+3 x+2 \\
-x^{3} & -x^{3}-\frac{3}{2} x^{2}-\frac{1}{2} x & -x^{3}-3 x^{2}-\frac{5}{2} x-\frac{1}{2} & -x^{3}-\frac{9}{2} x^{2}-6 x-\frac{9}{4}
\end{array}\right) \\
& =\left(\begin{array}{cccc}
1 & 1 & 1 & 1 \\
-x & -x-\frac{1}{2} & -x-1 & -x-\frac{3}{2} \\
x^{2}-x & x^{2}-\frac{1}{3} & x^{2}+x-\frac{1}{6} & x^{2}+2 x+\frac{1}{2} \\
3 x^{2}-x^{3}-2 x & \frac{3 x^{2}}{2}-x^{3}+\frac{x}{2}-\frac{1}{2} & \frac{3 x}{2}-x^{3} & x-\frac{3 x^{2}}{2}-x^{3}+\frac{3}{4}
\end{array}\right) .
\end{aligned}
$$


Kim et al. ([8], Theorem 11) introduced the following result: for $n \in \mathbb{Z}$ and $k \in \mathbb{N}$,

$$
B_{n}^{(k)}(-x)=\sum_{m=0}^{n}(-1)^{n-m} s_{2}(n, m) \hat{D}_{m}^{k}[(k)](x) .
$$

We can write Eq. (38) in the matrix form as follows:

$$
\mathbf{B}^{(k)}(-x)=\tilde{\mathbf{S}}_{2} \hat{\mathbf{D}}^{(k)}(x) .
$$

For example, setting $0 \leq n \leq 3$ and $0 \leq k \leq n$ in (39), we have

$$
\begin{aligned}
& \left(\begin{array}{cccc}
1 & 0 & 0 & 0 \\
0 & 1 & 0 & 0 \\
0 & -1 & 1 & 0 \\
0 & 1 & -3 & 1
\end{array}\right)\left(\begin{array}{cccc}
1 & 1 & 1 & 1 \\
-x & -x-\frac{1}{2} & -x-1 & -x-\frac{3}{2} \\
x^{2}-x & x^{2}-\frac{1}{3} & x^{2}+x-\frac{1}{6} & x^{2}+2 x+\frac{1}{2} \\
3 x^{2}-x^{3}-2 x & \frac{3 x^{2}}{2}-x^{3}+\frac{x}{2}-\frac{1}{2} & \frac{3 x}{2}-x^{3} & x-\frac{3 x^{2}}{2}-x^{3}+\frac{3}{4}
\end{array}\right) \\
& =\left(\begin{array}{cccc}
1 & 1 & 1 & -x-\frac{3}{2} \\
-x & -x-\frac{1}{2} & -x-1 & x^{2}+3 x+2 \\
x^{2} & x^{2}+x+\frac{1}{6} & x^{2}+2 x+\frac{5}{6} & -x^{3}-\frac{9 x^{2}}{2}-6 x-\frac{9}{4} \\
-x^{3} & -x^{3}-\frac{3 x^{2}}{2}-\frac{x}{2} & -x^{3}-3 x^{2}-\frac{5 x}{2}-\frac{1}{2} & -x^{2}
\end{array}\right.
\end{aligned}
$$

Remark 6 We can prove Eq. (39) ([8], Theorem 11), directly by using the matrix form (37) as follows. Multiplying both sides of (37) by $\tilde{\mathbf{S}}_{2}$, we get

$$
\tilde{\mathbf{S}}_{2} \hat{\mathbf{D}}^{(k)}(x)=\tilde{\mathbf{S}}_{2} \mathbf{S} \mathbf{B}^{(k)}(-x)=\mathbf{I B}^{(k)}(-x)=\mathbf{B}^{(k)}(-x),
$$

and, thus, we have Eq. (39).

\section{The $\lambda$-Daehee numbers and polynomials of higher order}

In this section we introduce the matrix representation for the $\lambda$-Daehee numbers and polynomials of higher order given by Kim et al. [9]. Hence, we can derive these results in matrix representation and prove these results simply by using the given matrix forms.

The $\lambda$-Daehee polynomials of the first kind of order $k$ can be defined by the generating function

$$
\left(\frac{\lambda \log (1+t)}{(1+t)^{\lambda}-1}\right)^{k}(1+t)^{x}=\sum_{n=0}^{\infty} D_{n, \lambda}^{(k)}(x) \frac{t^{n}}{n !}
$$

When $x=0, D_{n, \lambda}^{(k)}=D_{n, \lambda}^{(k)}(0)$ are called the $\lambda$-Daehee numbers of order $k$ :

$$
\left(\frac{\lambda \log (1+t)}{(1+t)^{\lambda}-1}\right)^{k}=\sum_{n=0}^{\infty} D_{n, \lambda}^{(k)} \frac{t^{n}}{n !}
$$

It is easy to see that $D_{n}^{(k)}(x)=D_{n, 1}^{(k)}(x)$ and $D_{n, \lambda}(x)=D_{n, \lambda}^{(1)}(x)$.

Kim et al. ([9], Theorem 3) obtained the following results: for $n \geq 0$ and $k \in \mathbb{N}$,

$$
D_{n, \lambda}^{(k)}(x)=\sum_{m=0}^{n} s_{1}(n, m) \lambda^{m} B_{m}^{(k)}\left(\frac{x}{\lambda}\right)
$$


and

$$
\lambda^{n} B_{n}^{(k)}\left(\frac{x}{\lambda}\right)=\sum_{m=0}^{n} s_{2}(n, m) D_{m, \lambda}^{(k)}(x)
$$

We can write these results in the following matrix forms:

$$
\mathbf{D}_{\lambda}^{(k)}(x)=\mathbf{S}_{1} \mathbf{\Lambda} \mathbf{B}^{(k)}\left(\frac{x}{\lambda}\right)
$$

and

$$
\boldsymbol{\Lambda} \mathbf{B}^{(k)}\left(\frac{x}{\lambda}\right)=\mathbf{S}_{2} \mathbf{D}_{\lambda}^{(k)}(x),
$$

where, $\mathbf{D}_{\lambda}^{(k)}(x)$ is the $(n+1) \times(k+1)$ matrix for the $\lambda$-Daehee polynomials of the first kind of order $k, \mathbf{B}^{(k)}(x / \lambda)$ is the $(n+1) \times(k+1)$ matrix for the Bernoulli polynomials of order $k$ with $x \rightarrow x / \lambda$, and $\boldsymbol{\Lambda}$ is the $(n+1) \times(n+1)$ diagonal matrix with elements $(\boldsymbol{\Lambda})_{i i}=\lambda^{i}$, $i=j=0,1, \ldots, n$.

For example, setting $0 \leq n \leq 3$ and $0 \leq k \leq n$ in (44), we have

$$
\begin{aligned}
& \left(\begin{array}{cccc}
1 & 0 & 0 & 0 \\
0 & 1 & 0 & 0 \\
0 & -1 & 1 & 0 \\
0 & 2 & -3 & 1
\end{array}\right)\left(\begin{array}{cccc}
1 & 0 & 0 & 0 \\
0 & \lambda & 0 & 0 \\
0 & 0 & \lambda^{2} & 0 \\
0 & 0 & 0 & \lambda^{3}
\end{array}\right) \\
& \times\left(\begin{array}{cccc}
1 & 1 & 1 & 1 \\
\frac{x}{\lambda} & \frac{x}{\lambda}-\frac{1}{2} & \frac{x}{\lambda}-1 & \frac{x}{\lambda}-\frac{3}{2} \\
\frac{x^{2}}{\lambda^{2}} & \frac{x^{2}}{\lambda^{2}}-\frac{x}{\lambda}+\frac{1}{6} & \frac{x^{2}}{\lambda^{2}}-\frac{2 x}{\lambda}+\frac{5}{6} & \frac{x^{2}}{\lambda^{2}}-\frac{3 x}{\lambda}+2 \\
\frac{x^{3}}{\lambda^{3}} & \frac{x}{2 \lambda}-\frac{3 x^{2}}{2 \lambda^{2}}+\frac{x^{3}}{\lambda^{3}} & \frac{5 x}{2 \lambda}-\frac{3 x^{2}}{\lambda^{2}}+\frac{x^{3}}{\lambda^{3}}-\frac{1}{2} & \frac{6 x}{\lambda}-\frac{9 x^{2}}{2 \lambda^{2}}+\frac{x^{3}}{\lambda^{3}}-\frac{9}{4}
\end{array}\right) \\
& =\left(\begin{array}{cccc}
1 & 1 & 1 & 1 \\
x & x-\frac{\lambda}{2} & x-\lambda & x-\frac{3 \lambda}{2} \\
x(x-1) & \frac{\lambda^{2}}{6}-\lambda x+\frac{\lambda}{2}+x^{2}-x & \frac{5 \lambda^{2}}{6}-2 \lambda x+\lambda+x^{2}-x & 2 \lambda^{2}-3 \lambda x+\frac{3 \lambda}{2}+x^{2}-x \\
D_{3, \lambda}^{(0)}(x) & D_{3, \lambda}^{(1)}(x) & D_{3, \lambda}^{(2)}(x) & D_{3, \lambda}^{(3)}(x)
\end{array}\right) \text {, }
\end{aligned}
$$

where

$$
\begin{aligned}
& D_{3, \lambda}^{(0)}(x)=x(x-1)(x-2), \quad D_{3, \lambda}^{(1)}(x)=-\frac{1}{2}(\lambda-2 x+2)\left(\lambda-2 x+x^{2}-\lambda x\right), \\
& D_{3, \lambda}^{(2)}(x)=-\frac{1}{2}(\lambda-x+1)\left(\lambda^{2}-4 \lambda x+4 \lambda+2 x^{2}-4 x\right), \\
& D_{3, \lambda}^{(3)}(x)=-\frac{1}{4}(3 \lambda-2 x+2)\left(3 \lambda^{2}-6 \lambda x+6 \lambda+2 x^{2}-4 x\right) .
\end{aligned}
$$

Remark 7 In fact, we can prove Eq. (45) by simply by multiplying Eq. (44) by $\mathbf{S}_{2}$ as follows:

$$
\mathbf{S}_{2} \mathbf{D}_{\lambda}^{(k)}(x)=\mathbf{S}_{2} \mathbf{S}_{1} \mathbf{\Lambda} \mathbf{B}^{(k)}\left(\frac{x}{\lambda}\right)=\mathbf{I} \mathbf{\Lambda} \mathbf{B}^{(k)}\left(\frac{x}{\lambda}\right)=\mathbf{\Lambda} \mathbf{B}^{(k)}\left(\frac{x}{\lambda}\right) .
$$

The following theorem gives a relation between the Daehee polynomials of higher order and $\lambda$-Daehee polynomials of higher order. 
Theorem 4 For $m \geq 0$, we have

$$
D_{m, \lambda}^{(k)}(\lambda x)=m ! \sum_{n=0}^{m} \sum_{i_{1}+i_{2}+\cdots+i_{n}=m} \frac{D_{n}^{(k)}(x)}{n !}\left(\begin{array}{l}
\lambda \\
i_{1}
\end{array}\right)\left(\begin{array}{l}
\lambda \\
i_{2}
\end{array}\right) \ldots\left(\begin{array}{c}
\lambda \\
i_{n}
\end{array}\right) .
$$

Proof From (16), replacing $(1+t)$ by $(1+t)^{\lambda}$, we have

$$
\left(\frac{\lambda \log (1+t)}{(1+t)^{\lambda}-1}\right)^{k}(1+t)^{\lambda x}=\sum_{n=0}^{\infty} D_{n}^{(k)}(x) \frac{\left((1+t)^{\lambda}-1\right)^{n}}{n !} .
$$

Thus, from (40) we get

$$
\begin{aligned}
\sum_{m=0}^{\infty} D_{m, \lambda}^{(k)}(\lambda x) \frac{t^{m}}{m !} & =\sum_{n=0}^{\infty} \frac{D_{n}^{(k)}(x)}{n !}\left(\sum_{i=0}^{\lambda}\left(\begin{array}{c}
\lambda \\
i
\end{array}\right) t^{i}-1\right)^{n} \\
& =\sum_{n=0}^{\infty} \frac{D_{n}^{(k)}(x)}{n !}\left(\sum_{i=1}^{\lambda}\left(\begin{array}{c}
\lambda \\
i
\end{array}\right) t^{i}\right)^{n} .
\end{aligned}
$$

Using the Cauchy rule for a product of series, we obtain

$$
\begin{aligned}
\sum_{m=0}^{\infty} D_{m, \lambda}^{(k)}(\lambda x) \frac{t^{m}}{m !} & =\sum_{n=0}^{\infty} \frac{D_{n}^{(k)}(x)}{n !} \sum_{m=n}^{\infty} \sum_{i_{1}+i_{2}+\cdots+i_{n}=m}\left(\begin{array}{l}
\lambda \\
i_{1}
\end{array}\right) \cdots\left(\begin{array}{l}
\lambda \\
i_{n}
\end{array}\right) t^{m} \\
& =\sum_{m=0}^{\infty} m ! \sum_{n=0}^{m} \sum_{i_{1}+i_{2}+\cdots+i_{n}=m} \frac{D_{n}^{(k)}(x)}{n !}\left(\begin{array}{l}
\lambda \\
i_{1}
\end{array}\right) \cdots\left(\begin{array}{c}
\lambda \\
i_{n}
\end{array}\right) \frac{t^{m}}{m !} .
\end{aligned}
$$

Equating the coefficients of $t^{m}$ on both sides yields (46). This completes the proof.

Setting $x=0$ in (46), we have the following corollary as a particular case.

Corollary 3 For $m \geq 0$, we have

$$
D_{m, \lambda}^{(k)}=m ! \sum_{n=0}^{m} \sum_{i_{1}+i_{2}+\cdots+i_{n}=m} \frac{D_{n}^{(k)}}{n !}\left(\begin{array}{l}
\lambda \\
i_{1}
\end{array}\right)\left(\begin{array}{l}
\lambda \\
i_{2}
\end{array}\right) \cdots\left(\begin{array}{l}
\lambda \\
i_{n}
\end{array}\right) .
$$

Kim et al. [9] defined the $\lambda$-Daehee polynomials of the second kind of order $k$ as follows:

$$
\left(\frac{\lambda \log (1+t)}{(1+t)^{\lambda}-1}\right)^{k}(1+t)^{\lambda k+x}=\sum_{n=0}^{\infty} \hat{D}_{n, \lambda}^{(k)}(x) \frac{t^{n}}{n !} .
$$

Kim et al. ([9], Theorem 5) proved that

$$
\hat{D}_{m, \lambda}^{(k)}(x)=\sum_{l=0}^{m} s_{1}(m, l) \lambda^{l} B_{l}^{(k)}\left(k+\frac{x}{\lambda}\right)
$$

and

$$
\lambda^{m} B_{m}^{(k)}\left(k+\frac{x}{\lambda}\right)=\sum_{n=0}^{m} s_{2}(m, n) \hat{D}_{n, \lambda}^{(k)}(x) .
$$


Also, Kim et al. ([9], Eq. (35)) introduced the following result:

$$
B_{n}^{(k)}(k-x)=(-1)^{n} B_{n}^{(k)}(x)
$$

Remark 8 We can write (49) and (50), respectively, in the following matrix forms:

$$
\hat{\mathbf{D}}_{\lambda}^{(k)}(x)=\mathbf{S}_{1} \mathbf{\Lambda}_{1} \mathbf{B}^{(k)}\left(-\frac{x}{\lambda}\right)
$$

and

$$
\mathbf{\Lambda}_{1} \mathbf{B}^{(k)}\left(-\frac{x}{\lambda}\right)=\mathbf{S}_{2} \hat{\mathbf{D}}_{\lambda}^{(k)}(x)
$$

where $\hat{\mathbf{D}}_{\lambda}(x)$ is the $(n+1) \times(n+1)$ matrix for the $\lambda$-Daehee polynomials of the second kind of order $k$, and $\boldsymbol{\Lambda}_{1}$ is the $(n+1) \times(n+1)$ diagonal matrix with elements $\left(\boldsymbol{\Lambda}_{1}\right)_{i i}=(-\lambda)^{i}$ for $i=j=0,1, \ldots, n$.

For example, setting $0 \leq n \leq 3$ and $0 \leq k \leq n$ in (52), we have

$$
\begin{aligned}
& \left(\begin{array}{cccc}
1 & 0 & 0 & 0 \\
0 & 1 & 0 & 0 \\
0 & -1 & 1 & 0 \\
0 & 2 & -3 & 1
\end{array}\right)\left(\begin{array}{cccc}
1 & 0 & 0 & 0 \\
0 & -\lambda & 0 & 0 \\
0 & 0 & \lambda^{2} & 0 \\
0 & 0 & 0 & -\lambda^{3}
\end{array}\right) \\
& \times\left(\begin{array}{cccc}
1 & 1 & 1 & 1 \\
-\frac{x}{\lambda} & -\frac{x}{\lambda}-\frac{1}{2} & -\frac{x}{\lambda}-1 & -\frac{x}{\lambda}-\frac{3}{2} \\
\frac{x^{2}}{\lambda^{2}} & \frac{1}{\lambda^{2}}\left(\frac{\lambda^{2}}{6}+\lambda x+x^{2}\right) & \frac{1}{\lambda^{2}}\left(\frac{5}{6} \lambda^{2}+2 \lambda x+x^{2}\right) & \frac{1}{\lambda^{2}}(2 \lambda+x)(\lambda+x) \\
-\frac{x^{3}}{\lambda^{3}} & \frac{x(\lambda+2 x)(\lambda+x)}{2 \lambda^{3}} & -\frac{(\lambda+x)\left(\lambda^{2}+4 \lambda x+2 x^{2}\right)}{2 \lambda^{3}} & -\frac{(3 \lambda+2 x)\left(3 \lambda^{2}+6 \lambda x+2 x^{2}\right)}{4 \lambda^{3}}
\end{array}\right) \\
& =\left(\begin{array}{cccc}
1 & 1 & 1 & 1 \\
x & \frac{\lambda}{2}+x & \lambda+x & \frac{3 \lambda}{2}+x \\
x(x-1) & \frac{\lambda^{2}}{6}+\lambda x-\frac{\lambda}{2}+x^{2}-x & \frac{5 \lambda^{2}}{6}+2 \lambda x-\lambda+x^{2}-x & 2 \lambda^{2}+3 \lambda x-\frac{3 \lambda}{2}+x^{2}-x \\
\hat{D}_{3, \lambda}^{(0)} & \hat{D}_{3, \lambda}^{(1)}(x) & \hat{D}_{3, \lambda}^{(2)}(x) & \hat{D}_{3, \lambda}^{(3)}(x)
\end{array}\right),
\end{aligned}
$$

where

$$
\begin{aligned}
& \hat{D}_{3, \lambda}^{(0)}(x)=x(x-1)(x-2), \quad \hat{D}_{3, \lambda}^{(1)}(x)=-\frac{1}{2}(\lambda+2 x-2)\left(\lambda+2 x-x^{2}-\lambda x\right), \\
& \hat{D}_{3, \lambda}^{(2)}(x)=\frac{1}{2}(\lambda+x-1)\left(\lambda^{2}+4 \lambda x-4 \lambda+2 x^{2}-4 x\right), \\
& \hat{D}_{3, \lambda}^{(3)}(x)=\frac{1}{4}(3 \lambda+2 x-2)\left(3 \lambda^{2}+6 \lambda x-6 \lambda+2 x^{2}-4 x\right) .
\end{aligned}
$$

Remark 9 We can prove Eq. (50) easily by using the matrix form, multiplying Eq. (52) by $\mathbf{S}_{2}$ as follows:

$$
\mathbf{S}_{2} \hat{\mathbf{D}}_{\lambda}^{(k)}(x)=\mathbf{S}_{2} \mathbf{S}_{1} \mathbf{\Lambda}_{1} \mathbf{B}^{(k)}\left(-\frac{x}{\lambda}\right)=\mathbf{I} \mathbf{\Lambda}_{1} \mathbf{B}^{(k)}\left(-\frac{x}{\lambda}\right)=\boldsymbol{\Lambda}_{1} \mathbf{B}^{(k)}\left(-\frac{x}{\lambda}\right) .
$$




\section{The twisted $\lambda$-Daehee numbers and polynomials of higher order}

Kim et al. [10] defined the twisted $\lambda$-Daehee polynomials of the first kind of order $k$ by the generating function

$$
\left(\frac{\lambda \log (1+\xi t)}{(1+\xi t)^{\lambda}-1}\right)^{k}(1+\xi t)^{x}=\sum_{n=0}^{\infty} D_{n, \xi}^{(k)}(x \mid \lambda) \frac{t^{n}}{n !} .
$$

In the special case $x=0, D_{n, \xi, \lambda}^{(k)}=D_{n, \xi}^{(k)}(0 \mid \lambda)$ are called the twisted $\lambda$-Daehee numbers of the first kind of order $k$ :

$$
\left(\frac{\lambda \log (1+\xi t)}{(1+\xi t)^{\lambda}-1}\right)^{k}=\sum_{n=0}^{\infty} D_{n, \xi, \lambda}^{(k)} \frac{t^{n}}{n !}
$$

The twisted Bernoulli polynomials of order $r \in \mathbb{N}$ are defined by the generating function (see [4])

$$
\left(\frac{t}{\xi e^{t}-1}\right)^{r} e^{x t}=\sum_{n=0}^{\infty} B_{n, \xi}^{(r)}(x) \frac{t^{n}}{n !}
$$

The relation between the twisted $\lambda$-Daehee polynomials and $\lambda$-Daehee polynomials of order $k$ is given in the following corollary.

Corollary 4 For $n \geq 0$ and $k \in \mathbb{N}$, we have

$$
D_{n, \xi}^{(k)}(x \mid \lambda)=\xi^{n} D_{n, \lambda}^{(k)}(x)
$$

Proof Replacing $t$ with $\xi t$ in (40), we have

$$
\left(\frac{\lambda \log (1+\xi t)}{(1+\xi t)^{\lambda}-1}\right)^{k}(1+\xi t)^{x}=\sum_{n=0}^{\infty} D_{n, \lambda}^{(k)}(x) \frac{(\xi t)^{n}}{n !}=\sum_{n=0}^{\infty} \xi^{n} D_{n, \lambda}^{(k)}(x) \frac{t^{n}}{n !}
$$

Hence, by virtue of (54) and (58) we get (57). This completes the proof.

If we put $x=0$ and $\lambda=1$ in (57), then we have, respectively,

$$
D_{n, \xi, \lambda}^{(k)}=\xi^{n} D_{n, \lambda}^{(k)} \quad \text { and } \quad D_{n, \xi}^{(k)}(x)=\xi^{n} D_{n}^{(k)}(x)
$$

Kim et al. ([10], Theorem 1) proved the following relation: for $m \in \mathbb{Z}$ and $k \in \mathbb{N}$,

$$
D_{m, \xi}^{(k)}(x \mid \lambda)=\xi^{m} \sum_{l=0}^{m} S_{1}(m, l) \lambda^{l} B_{l}^{(k)}\left(\frac{x}{\lambda}\right)
$$

and

$$
\lambda^{m} B_{m, \xi^{\lambda}}^{(k)}\left(\frac{x}{\lambda}\right)=\sum_{n=0}^{m} D_{n, \xi}^{(k)}(x \mid \lambda) \xi^{-n-x} s_{2}(m, n),
$$


where $B_{m, \xi^{\lambda}}^{(k)}\left(\frac{x}{\lambda}\right)$ is defined by Kim et al. ([10], Eq. (15)) as follows:

$$
\left(\frac{\lambda t}{\xi^{\lambda} e^{\lambda t}-1}\right)^{k}\left(\xi e^{t}\right)^{x}=\xi^{x} \sum_{m=0}^{\infty} \lambda^{m} B_{m, \xi^{\lambda}}^{(k)}\left(\frac{x}{\lambda}\right) \frac{t^{m}}{m !} .
$$

Remark 10 We can write (59) in the matrix form

$$
\mathbf{D}_{\xi}^{(k)}(x \mid \lambda)=\boldsymbol{\Xi} \mathbf{S}_{1} \mathbf{\Lambda} \mathbf{B}^{(k)}\left(\frac{x}{\lambda}\right)
$$

where $\mathbf{D}_{\xi}^{(k)}(x \mid \lambda)$ is the $(n+1) \times(k+1)$ matrix for the twisted Daehee numbers of the first kind of order $k$, and $\boldsymbol{\Xi}$ is the $(n+1) \times(n+1)$ diagonal matrix with elements $(\boldsymbol{\Xi})_{i i}=\xi^{i}$ for $i=j=0,1, \ldots, n$.

For example, setting $0 \leq n \leq 3$ and $0 \leq k \leq n$ in (62), we have

$$
\begin{aligned}
& \left(\begin{array}{llll}
1 & 0 & 0 & 0 \\
0 & \xi & 0 & 0 \\
0 & 0 & \xi^{2} & 0 \\
0 & 0 & 0 & \xi^{3}
\end{array}\right)\left(\begin{array}{cccc}
1 & 0 & 0 & 0 \\
0 & 1 & 0 & 0 \\
0 & -1 & 1 & 0 \\
0 & 2 & -3 & 1
\end{array}\right)\left(\begin{array}{cccc}
1 & 0 & 0 & 0 \\
0 & \lambda & 0 & 0 \\
0 & 0 & \lambda^{2} & 0 \\
0 & 0 & 0 & \lambda^{3}
\end{array}\right) \\
& \times\left(\begin{array}{cccc}
1 & 1 & 1 & 1 \\
\frac{x}{\lambda} & \frac{x}{\lambda}-\frac{1}{2} & \frac{x}{\lambda}-1 & \frac{x}{\lambda}-\frac{3}{2} \\
\frac{x^{2}}{\lambda^{2}} & \frac{x^{2}}{\lambda^{2}}-\frac{x}{\lambda}+\frac{1}{6} & \frac{x^{2}}{\lambda^{2}}-\frac{2 x}{\lambda}+\frac{5}{6} & \frac{x^{2}}{\lambda^{2}}-\frac{3 x}{\lambda}+2 \\
\frac{x^{3}}{\lambda^{3}} & \frac{x}{2 \lambda}-\frac{3 x^{2}}{2 \lambda^{2}}+\frac{x^{3}}{\lambda^{3}} & \frac{5 x}{2 \lambda}-\frac{3 x^{2}}{\lambda^{2}}+\frac{x^{3}}{\lambda^{3}}-\frac{1}{2} & \frac{6 x}{\lambda}-\frac{9 x^{2}}{2 \lambda^{2}}+\frac{x^{3}}{\lambda^{3}}-\frac{9}{4}
\end{array}\right) \\
& =\left(\begin{array}{cccc}
1 & 1 & 1 & 1 \\
\xi x & -\frac{\xi}{2}(\lambda-2 x) & -\xi(\lambda-x) & -\frac{\xi}{2}(3 \xi-2 x) \\
\xi^{2} x(x-1) & \xi^{2}\left(\frac{\lambda^{2}}{6}-\lambda x+\frac{\lambda}{2}+x^{2}-x\right) & \xi^{2}\left(\frac{5}{6} \lambda^{2}-2 \lambda x+\lambda+x^{2}-x\right) & \xi^{2}\left(2 \lambda^{2}-3 \lambda x+\frac{3}{2} \lambda+x^{2}-x\right) \\
D_{3, \xi}^{(0)}(x \mid \lambda) & D_{3, \xi}^{(1)}(x \mid \lambda) & D_{3, \xi}^{(2)}(x \mid \lambda) & D_{3, \xi}^{(3)}(x \mid \lambda)
\end{array}\right),
\end{aligned}
$$

where

$$
\begin{aligned}
& D_{3, \xi}^{(0)}(x \mid \lambda)=\xi^{3} x(x-1)(x-2), \quad D_{3, \xi}^{(1)}(x \mid \lambda)=-\frac{\xi^{3}}{2}(\lambda-2 x+2)\left(\lambda-2 x+x^{2}-\lambda x\right), \\
& D_{3, \xi}^{(2)}(x \mid \lambda)=-\frac{\xi^{3}}{2}(\lambda-x+1)\left(\lambda^{2}-4 \lambda x+4 \lambda+2 x^{2}-4 x\right), \\
& D_{3, \xi}^{(0)}(x \mid \lambda)=-\frac{\xi^{3}}{4}(3 \lambda-2 x+2)\left(3 \lambda^{2}-6 \lambda x+6 \lambda+2 x^{2}-4 x\right) .
\end{aligned}
$$

Remark 11 In fact, it seems that the statement in (60) is not correct (the second equation of Kim et al. [10], Theorem 1). From (62), multiplying both sides by $\boldsymbol{\Xi}^{-1}$, we have

$$
\boldsymbol{\Xi}^{-1} \mathbf{D}_{\xi}^{(k)}(x \mid \lambda)=\boldsymbol{\Xi}^{-1} \boldsymbol{\Xi} \mathbf{S}_{1} \mathbf{\Lambda} \mathbf{B}^{(k)}\left(\frac{x}{\lambda}\right)=\mathbf{S}_{1} \mathbf{\Lambda} \mathbf{B}^{(k)}\left(\frac{x}{\lambda}\right)
$$

then multiplying both sides by $\mathbf{S}_{2}$, we have

$$
\mathbf{S}_{2} \boldsymbol{\Xi}^{-1} \mathbf{D}_{\xi}^{(k)}(x \mid \lambda)=\mathbf{S}_{2} \mathbf{S}_{1} \mathbf{\Lambda} \mathbf{B}^{(k)}\left(\frac{x}{\lambda}\right)=\mathbf{\Lambda} \mathbf{B}^{(k)}\left(\frac{x}{\lambda}\right) .
$$

From (60) and (63) it is clear that there is a contradiction. 
In the following theorem we obtained a corrected relation.

Theorem 5 For $m \in \mathbb{Z}$ and $k \in \mathbb{N}$, we have

$$
\lambda^{m} B_{m}^{(k)}\left(\frac{x}{\lambda}\right)=\sum_{n=0}^{m} D_{n, \xi}^{(k)}(x \mid \lambda) \xi^{-n} s_{2}(m, n) .
$$

Proof From Eq. (54), replacing $t$ by $\left(e^{t}-1\right) / \xi$, we have

$$
\begin{aligned}
& \left(\frac{\lambda \log \left(1+\frac{\xi\left(e^{t}-1\right)}{\xi}\right)}{\left(1+\frac{\xi\left(e^{t}-1\right)}{\xi}\right)^{\lambda}-1}\right)^{k}\left(1+\frac{\xi\left(e^{t}-1\right)}{\xi}\right)^{x}=\sum_{n=0}^{\infty} D_{n, \xi}^{(k)}(x \mid \lambda) \frac{\left(e^{t}-1\right)^{n}}{n ! \xi^{n}} \\
& \left(\frac{\lambda t}{e^{\lambda t}-1}\right)^{k} e^{t x}=\sum_{n=0}^{\infty} D_{n, \xi}^{(k)}(x \mid \lambda) \frac{\left(e^{t}-1\right)^{n}}{n ! \xi^{n}}
\end{aligned}
$$

Substituting (7) into (65), we have

$$
\begin{aligned}
\left(\frac{\lambda t}{e^{\lambda t}-1}\right)^{k} e^{\lambda t\left(\frac{x}{\lambda}\right)} & =\sum_{n=0}^{\infty} D_{n, \xi}^{(k)}(x \mid \lambda) \xi^{-n} \sum_{m=n}^{\infty} s_{2}(m, n) \frac{t^{m}}{m !} \\
& =\sum_{m=0}^{\infty} \sum_{n=0}^{m} D_{n, \xi}^{(k)}(x \mid \lambda) \xi^{-n} s_{2}(m, n) \frac{t^{m}}{m !}
\end{aligned}
$$

From (1) and (66) we have

$$
\sum_{m=0}^{\infty} \lambda^{m} B_{m}^{(k)}\left(\frac{x}{\lambda}\right) \frac{t^{m}}{m !}=\sum_{m=0}^{\infty} \sum_{n=0}^{m} D_{n, \xi}^{(k)}(x \mid \lambda) \xi^{-n} s_{2}(m, n) \frac{t^{m}}{m !} .
$$

Equating the coefficients of $t^{m}$ on both sides gives (64). This completes the proof.

Moreover, we can represent Eq. (64) in the following matrix form as in (63):

$$
\mathbf{B}^{(k)}\left(\frac{x}{\lambda}\right)=\boldsymbol{\Lambda}^{-1} \mathbf{S}_{2} \boldsymbol{\Xi}^{-1} \mathbf{D}_{\xi}^{(k)}(x \mid \lambda) .
$$

For example, setting $0 \leq n \leq 3$ and $0 \leq k \leq n$ in (68), we have

$$
\begin{aligned}
&\left(\begin{array}{llll}
1 & 0 & 0 & 0 \\
0 & \frac{1}{\lambda} & 0 & 0 \\
0 & 0 & \frac{1}{\lambda^{2}} & 0 \\
0 & 0 & 0 & \frac{1}{\lambda^{3}}
\end{array}\right)\left(\begin{array}{llll}
1 & 0 & 0 & 0 \\
0 & 1 & 0 & 0 \\
0 & 1 & 1 & 0 \\
0 & 1 & 3 & 1
\end{array}\right)\left(\begin{array}{cccc}
1 & 0 & 0 & 0 \\
0 & \frac{1}{\xi} & 0 & 0 \\
0 & 0 & \frac{1}{\xi^{2}} & 0 \\
0 & 0 & 0 & \frac{1}{\xi^{3}}
\end{array}\right) \\
& \times\left(\begin{array}{cccc}
1 & 1 & 1 & 1 \\
\xi x & -\frac{\xi}{2}(\lambda-2 x) & -\xi(\lambda-x) & -\frac{\xi}{2}(3 \xi-2 x) \\
\xi^{2} x(x-1) & \xi^{2}\left(\frac{\lambda^{2}}{6}-\lambda x+\frac{\lambda}{2}+x^{2}-x\right) & \xi^{2}\left(\frac{5}{6} \lambda^{2}-2 \lambda x+\lambda+x^{2}-x\right) & \xi^{2}\left(2 \lambda^{2}-3 \lambda x+\frac{3}{2} \lambda+x^{2}-x\right) \\
D_{3, \xi}^{(0)}(x \mid \lambda) & D_{3, \xi}^{(1)}(x \mid \lambda) & D_{3, \xi}^{(3)}(x \mid \lambda)
\end{array}\right) \\
&=\left(\begin{array}{cccc}
1 & 1 & \frac{x}{\lambda}-1 & 1 \\
\frac{x}{\lambda} & \frac{x}{\lambda}-\frac{1}{2} & \frac{x^{2}}{\lambda^{2}}-\frac{2 x}{\lambda}+\frac{5}{6} & \frac{x^{2}}{\lambda^{2}}-\frac{3 x}{\lambda}+2 \\
\frac{x^{2}}{\lambda^{2}} & \frac{x^{2}}{\lambda^{2}}-\frac{x}{\lambda}+\frac{1}{6} & \frac{5}{2} \\
\frac{x^{3}}{\lambda^{3}} & \frac{x}{2 \lambda}-\frac{3 x^{2}}{2 \lambda^{2}}+\frac{x^{3}}{\lambda^{3}} & \frac{5 x}{2 \lambda}-\frac{3 x^{2}}{\lambda^{2}}+\frac{x^{3}}{\lambda^{3}}-\frac{1}{2} & \frac{6 x}{\lambda}-\frac{9 x^{2}}{2 \lambda^{2}}+\frac{x^{3}}{\lambda^{3}}-\frac{9}{4}
\end{array}\right)
\end{aligned}
$$


where

$$
\begin{aligned}
& D_{3, \xi}^{(0)}(x \mid \lambda)=\xi^{3} x(x-1)(x-2), \quad D_{3, \xi}^{(1)}(x \mid \lambda)=-\frac{\xi^{3}}{2}(\lambda-2 x+2)\left(\lambda-2 x+x^{2}-\lambda x\right), \\
& D_{3, \xi}^{(2)}(x \mid \lambda)=-\frac{\xi^{3}}{2}(\lambda-x+1)\left(\lambda^{2}-4 \lambda x+4 \lambda+2 x^{2}-4 x\right), \\
& D_{3, \xi}^{(3)}(x \mid \lambda)=-\frac{\xi^{3}}{4}(3 \lambda-2 x+2)\left(3 \lambda^{2}-6 \lambda x+6 \lambda+2 x^{2}-4 x\right) .
\end{aligned}
$$

Kim et al. [10] introduced the twisted $\lambda$-Daehee polynomials of the second kind of order $k$ as follows:

$$
\left(\frac{\lambda \log (1+\xi t)(1+\xi t)^{\lambda}}{(1+\xi t)^{\lambda}-1}\right)^{k}(1+\xi t)^{x}=\sum_{n=0}^{\infty} \hat{D}_{n, \xi}^{(k)}(x \mid \lambda) \frac{t^{n}}{n !} .
$$

Setting $x=0, \hat{D}_{n, \xi, \lambda}^{(k)}=\hat{D}_{n, \xi}^{(k)}(0 \mid \lambda)$, we have the twisted Daehee numbers of second kind of order $k$ :

$$
\left(\frac{\lambda \log (1+\xi t)(1+\xi t)^{\lambda}}{(1+\xi t)^{\lambda}-1}\right)^{k}=\sum_{n=0}^{\infty} \hat{D}_{n, \xi, \lambda}^{(k)} \frac{t^{n}}{n !}
$$

Kim et al. ([10], Theorem 2) proved that, for $m \in \mathbb{Z}$ and $k \in \mathbb{N}$,

$$
\xi^{-m} \hat{D}_{n, \xi}(x \mid \lambda)=\sum_{l=0}^{m} s_{1}(m, l) \lambda^{l} B_{l}^{(k)}\left(k+\frac{x}{\lambda}\right)
$$

and

$$
\lambda^{m} B_{m, \xi^{\lambda}}^{(k)}\left(k+\frac{x}{\lambda}\right)=\sum_{n=0}^{m} \hat{D}_{n, \xi}^{(k)}(x \mid \lambda) s_{2}(m, n) \xi^{-n-\lambda k-x}
$$

Using Eq. (51), we can write (71) in the matrix form

$$
\hat{\mathbf{D}}_{\xi}^{(k)}(x \mid \lambda)=\mathbf{\Xi} \mathbf{S}_{1} \mathbf{\Lambda}_{1} \mathbf{B}^{(k)}\left(-\frac{x}{\lambda}\right),
$$

where $\hat{\mathbf{D}}_{\xi}^{(k)}(x \mid \lambda)$ is the $(n+1) \times(k+1)$ matrix for the twisted Daehee numbers of the second kind of order $k$.

For example, setting $0 \leq n \leq 3$ and $0 \leq k \leq n$ in (73), we have

$$
\begin{aligned}
&\left(\begin{array}{llll}
1 & 0 & 0 & 0 \\
0 & \xi & 0 & 0 \\
0 & 0 & \xi^{2} & 0 \\
0 & 0 & 0 & \xi^{3}
\end{array}\right)\left(\begin{array}{cccc}
1 & 0 & 0 & 0 \\
0 & 1 & 0 & 0 \\
0 & -1 & 1 & 0 \\
0 & 2 & -3 & 1
\end{array}\right)\left(\begin{array}{cccc}
1 & 0 & 0 & 0 \\
0 & -\lambda & 0 & 0 \\
0 & 0 & \lambda^{2} & 0 \\
0 & 0 & 0 & -\lambda^{3}
\end{array}\right) \\
& \times\left(\begin{array}{cccc}
1 & 1 & 1 & 1 \\
-\frac{x}{\lambda} & -\frac{x}{\lambda}-\frac{1}{2} & -\frac{x}{\lambda}-1 & -\frac{x}{\lambda}-\frac{3}{2} \\
\frac{x^{2}}{\lambda^{2}} & \frac{x^{2}}{\lambda^{2}}+\frac{x}{\lambda}+\frac{1}{6} & \frac{x^{2}}{\lambda^{2}}+\frac{2 x}{\lambda}+\frac{5}{6} & \frac{x^{2}}{\lambda^{2}}+\frac{3 x}{\lambda}+2 \\
-\frac{x^{3}}{\lambda^{3}} & -\frac{x}{2 \lambda}-\frac{3 x^{2}}{2 \lambda^{2}}-\frac{x^{3}}{\lambda^{3}} & -\frac{5 x}{2 \lambda}-\frac{3 x^{2}}{\lambda^{2}}-\frac{x^{3}}{\lambda^{3}}-\frac{1}{2} & -\frac{6 x}{\lambda}-\frac{9 x^{2}}{2 \lambda^{2}}-\frac{x^{3}}{\lambda^{3}}-\frac{9}{4}
\end{array}\right)
\end{aligned}
$$




$$
=\left(\begin{array}{cccc}
1 & 1 & 1 & 1 \\
\xi x & \frac{\xi}{2}(\lambda+2 x) & \xi(\lambda+x) & \frac{\xi}{2}(3 \xi+2 x) \\
\xi^{2} x(x-1) & \xi^{2}\left(\frac{\lambda^{2}}{6}+\lambda x-\frac{\lambda}{2}+x^{2}-x\right) & \xi^{2}\left(\frac{5}{6} \lambda^{2}+2 \lambda x-\lambda+x^{2}-x\right) & \xi^{2}\left(2 \lambda^{2}+3 \lambda x-\frac{3}{2} \lambda+x^{2}-x\right) \\
\hat{D}_{3, \xi}^{(0)}(x \mid \lambda) & \hat{D}_{3, \xi}^{(1)}(x \mid \lambda) & \hat{D}_{3, \xi}^{(2)}(x \mid \lambda) & \hat{D}_{3, \xi}^{(3)}(x \mid \lambda)
\end{array}\right)
$$

where

$$
\begin{aligned}
& \hat{D}_{3, \xi}^{(0)}(x \mid \lambda)=\xi^{3} x(x-1)(x-2), \\
& \hat{D}_{3, \xi}^{(1)}(x \mid \lambda)=-\frac{\xi^{3}}{2}(\lambda+2 x-2)\left(\lambda+2 x-x^{2}-\lambda x\right), \\
& \hat{D}_{3, \xi}^{(2)}(x \mid \lambda)=\frac{\xi^{3}}{2}(\lambda+x-1)\left(\lambda^{2}+4 \lambda x-4 \lambda+2 x^{2}-4 x\right), \\
& \hat{D}_{3, \xi}^{(3)}(x \mid \lambda)=\frac{\xi^{3}}{4}(3 \lambda+2 x-2)\left(3 \lambda^{2}+6 \lambda x-6 \lambda+2 x^{2}-4 x\right) .
\end{aligned}
$$

From (73), multiplying both sides by $\boldsymbol{\Xi}^{-1}$, we have

$$
\boldsymbol{\Xi}^{-1} \hat{\mathbf{D}}_{\xi}^{(k)}(x \mid \lambda)=\boldsymbol{\Xi}^{-1} \boldsymbol{\Xi} \mathbf{S}_{1} \boldsymbol{\Lambda}_{1} \mathbf{B}^{(k)}\left(-\frac{x}{\lambda}\right)=\mathbf{S}_{1} \boldsymbol{\Lambda}_{1} \mathbf{B}^{(k)}\left(-\frac{x}{\lambda}\right)
$$

and multiplying both sides by $\mathbf{S}_{2}$, we have

$$
\mathbf{S}_{2} \boldsymbol{\Xi}^{-1} \hat{\mathbf{D}}_{\xi}^{(k)}(x \mid \lambda)=\mathbf{S}_{2} \mathbf{S}_{1} \boldsymbol{\Lambda}_{1} \mathbf{B}^{(k)}\left(-\frac{x}{\lambda}\right)=\mathbf{I} \boldsymbol{\Lambda}_{1} \mathbf{B}^{(k)}\left(-\frac{x}{\lambda}\right)=\boldsymbol{\Lambda}_{1} \mathbf{B}^{(k)}\left(-\frac{x}{\lambda}\right)
$$

From (72) and (74) we have a contradiction.

Remark 12 In fact, it clear that (72) is not correct (the second equation of Kim et al. [10], Theorem 2).

We give the correction of (72) in the following theorem.

Theorem 6 For $m \in \mathbb{Z}$ and $k \in \mathbb{N}$, we have

$$
\lambda^{m} B_{m}^{(k)}\left(k+\frac{x}{\lambda}\right)=\sum_{n=0}^{m} \hat{D}_{n, \xi}^{(k)}(x \mid \lambda) \xi^{-n} s_{2}(m, n)
$$

Proof From Eq. (69), replacing $t$ by $\left(e^{t}-1\right) / \xi$, we have

$$
\begin{aligned}
& \left(\frac{\lambda \log \left(1+\frac{\xi\left(e^{t}-1\right)}{\xi}\right)\left(1+\frac{\xi\left(e^{t}-1\right)}{\xi}\right)^{\lambda}}{\left(1+\frac{\xi\left(e^{t}-1\right)}{\xi}\right)^{\lambda}-1}\right)^{k}\left(1+\frac{\xi\left(e^{t}-1\right)}{\xi}\right)^{x}=\sum_{n=0}^{\infty} \hat{D}_{n, \xi}^{(k)}(x \mid \lambda) \frac{\left(e^{t}-1\right)^{n}}{n ! \xi^{n}} \\
& \left(\frac{\lambda t}{e^{\lambda t}-1}\right)^{k} e^{(k \lambda+x) t}=\sum_{n=0}^{\infty} \hat{D}_{n, \xi}^{(k)}(x \mid \lambda) \frac{\left(e^{t}-1\right)^{n}}{n ! \xi^{n}} \\
& \left(\frac{\lambda t}{e^{\lambda t}-1}\right)^{k} e^{\lambda t\left(k+\frac{x}{\lambda}\right)}=\sum_{n=0}^{\infty} \hat{D}_{n, \xi}^{(k)}(x \mid \lambda) \xi^{-n} \frac{\left(e^{t}-1\right)^{n}}{n !}
\end{aligned}
$$


Substituting Eq. (7) into Eq. (76), we have

$$
\begin{aligned}
\left(\frac{\lambda t}{e^{\lambda t}-1}\right)^{k} e^{\lambda t\left(k+\frac{x}{\lambda}\right)} & =\sum_{n=0}^{\infty} \hat{D}_{n, \xi}^{(k)}(x \mid \lambda) \xi^{-n} \sum_{m=n}^{\infty} s_{2}(m, n) \frac{t^{m}}{m !} \\
& =\sum_{m=0}^{\infty} \sum_{n=0}^{m} \hat{D}_{n, \xi}^{(k)}(x \mid \lambda) \xi^{-n} s_{2}(m, n) \frac{t^{m}}{m !}
\end{aligned}
$$

From Eq. (1) and Eq. (77) we have

$$
\sum_{m=0}^{\infty} \lambda^{m} B_{m}^{(k)}\left(k+\frac{x}{\lambda}\right) \frac{t^{m}}{m !}=\sum_{m=0}^{\infty} \sum_{n=0}^{m} \hat{D}_{n, \xi}^{(k)}(x \mid \lambda) \xi^{-n} s_{2}(m, n) \frac{t^{m}}{m !}
$$

Equating the coefficients of $t^{m}$ on both sides gives (75). This completes the proof.

Moreover, by using Eq. (51) we can represent Eq. (75) in the following matrix form:

$$
\mathbf{B}^{(k)}\left(-\frac{x}{\lambda}\right)=\boldsymbol{\Lambda}_{1}^{-1} \mathbf{S}_{2} \boldsymbol{\Xi}^{-1} \hat{\mathbf{D}}_{\xi}^{(k)}(x \mid \lambda),
$$

where $\boldsymbol{\Lambda} \mathbf{B}^{(k)}\left(k+\frac{x}{\lambda}\right)=\boldsymbol{\Lambda}_{1} \mathbf{B}^{(k)}\left(-\frac{x}{\lambda}\right)$.

For example, setting $0 \leq n \leq 3$ and $0 \leq k \leq n$ in (79), we have

$$
\begin{aligned}
& \left(\begin{array}{cccc}
1 & 0 & 0 & 0 \\
0 & -\frac{1}{\lambda} & 0 & 0 \\
0 & 0 & \frac{1}{\lambda^{2}} & 0 \\
0 & 0 & 0 & -\frac{1}{\lambda^{3}}
\end{array}\right)\left(\begin{array}{cccc}
1 & 0 & 0 & 0 \\
0 & 1 & 0 & 0 \\
0 & 1 & 1 & 0 \\
0 & 1 & 3 & 1
\end{array}\right)\left(\begin{array}{cccc}
1 & 0 & 0 & 0 \\
0 & \frac{1}{\xi} & 0 & 0 \\
0 & 0 & \frac{1}{\xi^{2}} & 0 \\
0 & 0 & 0 & \frac{1}{\xi^{3}}
\end{array}\right) \\
& \times\left(\begin{array}{cccc}
1 & 1 & 1 & 1 \\
\xi x & \frac{\xi}{2}(\lambda+2 x) & \xi(\lambda+x) & \frac{\xi}{2}(3 \xi+2 x) \\
\xi^{2} x(x-1) & \xi^{2}\left(\frac{\lambda^{2}}{6}+\lambda x-\frac{\lambda}{2}+x^{2}-x\right) & \xi^{2}\left(\frac{5}{6} \lambda^{2}+2 \lambda x-\lambda+x^{2}-x\right) & \xi^{2}\left(2 \lambda^{2}+3 \lambda x-\frac{3}{2} \lambda+x^{2}-x\right) \\
\hat{D}_{3, \xi}^{(0)}(x \mid \lambda) & \hat{D}_{3, \xi}^{(1)}(x \mid \lambda) & \hat{D}_{3, \xi}^{(2)}(x \mid \lambda) & \hat{D}_{3, \xi}^{(3)}(x \mid \lambda)
\end{array}\right) \\
& =\left(\begin{array}{cccc}
1 & 1 & 1 & 1 \\
-\frac{x}{\lambda} & -\frac{x}{\lambda}-\frac{1}{2} & -\frac{x}{\lambda}-1 & -\frac{x}{\lambda}-\frac{3}{2} \\
\frac{x^{2}}{\lambda^{2}} & \frac{x^{2}}{\lambda^{2}}+\frac{x}{\lambda}+\frac{1}{6} & \frac{x^{2}}{\lambda^{2}}+\frac{2 x}{\lambda}+\frac{5}{6} & \frac{x^{2}}{\lambda^{2}}+\frac{3 x}{\lambda}+2 \\
-\frac{x^{3}}{\lambda^{3}} & -\frac{x}{2 \lambda}-\frac{3 x^{2}}{2 \lambda^{2}}-\frac{x^{3}}{\lambda^{3}} & -\frac{5 x}{2 \lambda}-\frac{3 x^{2}}{\lambda^{2}}-\frac{x^{3}}{\lambda^{3}}-\frac{1}{2} & -\frac{6 x}{\lambda}-\frac{9 x^{2}}{2 \lambda^{2}}-\frac{x^{3}}{\lambda^{3}}-\frac{9}{4}
\end{array}\right) \text {, }
\end{aligned}
$$

where

$$
\begin{aligned}
& \hat{D}_{3, \xi}^{(0)}(x \mid \lambda)=\xi^{3} x(x-1)(x-2), \\
& \hat{D}_{3, \xi}^{(1)}(x \mid \lambda)=-\frac{\xi^{3}}{2}(\lambda+2 x-2)\left(\lambda+2 x-x^{2}-\lambda x\right), \\
& \hat{D}_{3, \xi}^{(2)}(x \mid \lambda)=\frac{\xi^{3}}{2}(\lambda+x-1)\left(\lambda^{2}+4 \lambda x-4 \lambda+2 x^{2}-4 x\right), \\
& \hat{D}_{3, \xi}^{(3)}(x \mid \lambda)=\frac{\xi^{3}}{4}(3 \lambda+2 x-2)\left(3 \lambda^{2}+6 \lambda x-6 \lambda+2 x^{2}-4 x\right) .
\end{aligned}
$$

For more details and very recent results on these numbers, see [17]. 


\section{Conclusions}

We derived generalization of higher-order Daehee numbers and polynomials. Moreover, new matrix representations for these numbers and polynomials are obtained. This enabled us to obtain simple and short proofs of many previous results on higher-order Daehee numbers and polynomials. Furthermore, we investigated the relations between these numbers and polynomials and Stirling numbers, Nörlund numbers, and Bernoulli numbers of higher-order. Finally, some numerical results are given.

\section{Appendix}

Mathcad program for higher-order Daehee numbers using the recurrence relation (11):

$$
D(n, k):=\mid \begin{aligned}
& D_{0,0} \leftarrow 1 \\
& \text { for } i \in 1 \cdots n \\
& D_{i, 0} \leftarrow 0 \\
& \text { for } j \in 1 \cdots k \\
& D_{0, j} \leftarrow 1 \\
& \text { for } i \in 0 \cdots n-1 \\
& \quad \text { for } j \in 1 \cdots k \\
& \quad D_{i+1, j+1} \leftarrow\left(\frac{1}{i+j+1}\right) \cdot\left[j \cdot D_{i+1, j-1}-(i+1) \cdot(i+j) \cdot D_{i, j}\right] \\
& D .
\end{aligned}
$$

\section{Competing interests}

The authors declare that they have no competing interests.

\section{Authors' contributions}

The authors, BSE-D and AM with the consultation of each other carried out this work and drafted the manuscript together. Both authors read and approved the final manuscript.

\section{Acknowledgements}

The authors would like to thank the referees for their valuable comments and suggestions.

Received: 26 October 2015 Accepted: 19 January 2016 Published online: 01 February 2016

\section{References}

1. El-Desouky, BS, Mustafa, A: New results and matrix representation for Daehee and Bernoulli numbers and polynomials. Appl. Math. Sci. 9(73), 3593-3610 (2015). doi:10.12988/ams.2015.53282

2. Carlitz, L: A note on Bernoulli and Euler polynomials of the second kind. Scr. Math. 25, 323-330 (1961)

3. Comtet, L: Advanced Combinatorics. Reidel, Dordrecht (1974)

4. Dolgy, DV, Kim, T, Lee, B, Lee, SH: Some new identities on the twisted Bernoulli and Euler polynomials. J. Comput. Anal. Appl. 14(3), 441-451 (2013)

5. El-Desouky, BS: The multiparameter non-central Stirling numbers. Fibonacci Q. 32(3), 218-225 (1994)

6. El-Desouky, BS, Cakić, NP, Mansour, T: Modified approach to generalized Stirling numbers via differential operators. Appl. Math. Lett. 23, 115-120 (2010)

7. Gould, HW: Explicit formulas for Bernoulli numbers. Am. Math. Mon. 79, 44-51 (1972)

8. Kim, DS, Kim, T, Lee, SH, Seo, Jj: Higher-order Daehee numbers and polynomials. Int. J. Math. Anal. 8(6), 273-283 (2014). doi:10.12988/ijma.2014.4118

9. Kim, DS, Kim, T, Lee, SH, Seo, JJ: A note on the lambda Daehee polynomials. Int. J. Math. Anal. 7(62), 3069-3080 (2013). doi:10.12988/ijma.2013.311264

10. Kim, DS, Kim, T, Lee, SH, Seo, JJ: A note on twisted $\lambda$-Daehee polynomials. Appl. Math. Sci. 7(141), 7005-7014 (2013). doi:10.12988/ams.2013.311635

11. Kim, DS, Kim, T: Daehee numbers and polynomials. Appl. Math. Sci. 7(120), 5969-5976 (2013). doi:10.12988/ams.2013.39535

12. Kim, T, Simsek, Y: Analytic continuation of the multiple Daehee $q$-l-functions associated with Daehee numbers. Russ. J. Math. Phys. 15, 58-65 (2008)

13. Kimura, N: On universal higher order Bernoulli numbers and polynomials. Report of the research, Institute of Industrial Technology, Nihon University, Number 70 (2003). ISSN:0386-1678 
14. Liu, GD, Srivastava, HM: Explicit formulas for the Nörlund polynomials $B_{n}^{(x)}$ and $b_{n}^{(x)}$. Comput. Math. Appl. 51, 1377-1384 (2006)

15. Ozden, H, Cangul, N, Simsek, Y: Remarks on $q$-Bernoulli numbers associated with Daehee numbers. Adv. Stud. Contemp. Math. (Kyungshang) 18, 41-48 (2009)

16. Wang, W: Generalized higher order Bernoulli number pairs and generalized Stirling number pairs. J. Math. Anal. Appl. 364, 255-274 (2010)

17. Araci, S, Agyuz, E, Acikgoz, M: On a q-analog of some numbers and polynomials. J. Inequal. Appl. 2015, 19 (2015). doi:10.1186/s13660-014-0542-y

Submit your manuscript to a SpringerOpen ${ }^{\circ}$ journal and benefit from:

- Convenient online submission

- Rigorous peer review

- Immediate publication on acceptance

- Open access: articles freely available online

- High visibility within the field

- Retaining the copyright to your article 